\title{
On the Routing in Flying Ad hoc Networks
}

\author{
Md. Hasan Tareque, Md. Shohrab Hossain \\ Department of Computer Science and Engineering \\ Bangladesh University of Engineering and Technology \\ Dhaka, Bangladesh \\ Email: hasantareque07@gmail.com, mshohrabhossain@cse.buet.ac.bd
}

\author{
Mohammed Atiquzzaman \\ School of Computer Science \\ The University of Oklahoma \\ Norman, OK 73019, USA \\ Email: atiq@ou.edu
}

\begin{abstract}
The usage of Unmanned Aerial Vehicles (UAVs) is increasing day by day. In recent years, UAVs are being used in increasing number of civil applications, such as policing, firefighting, etc in addition to military applications. Instead of using one large UAV, multiple UAVs are nowadays used for higher coverage area and accuracy. Therefore, networking models are required to allow two or more UAV nodes to communicate directly or via relay node(s). Flying Ad-Hoc Networks (FANETs) are formed which is basically an ad hoc network for UAVs. This is relatively a new technology in network family where requirements vary largely from traditional networking model, such as Mobile Ad-hoc Networks and Vehicular Ad-hoc Networks. In this paper, Flying Ad-Hoc Networks are surveyed along with its challenges compared to traditional ad hoc networks. The existing routing protocols for FANETs are then classified into six major categories which are critically analyzed and compared based on various performance criteria. Our comparative analysis will help network engineers in choosing appropriate routing protocols based on the specific scenario where the FANET will be deployed.
\end{abstract}

Index Terms-UAV networks, MANET, VANET, FANET, Routing protocols

\section{INTRODUCTION}

$\mathbf{U}$ NMANNED aerial vehicle (UAV) systems can fly independently or can be operated distantly. The usage of UAVs is increasing day by day. Earlier, UAVs were simple remotely piloted aircrafts and mostly used for military operations / applications. However, in recent years, UAVs are being used in increasing number of civil applications, such as policing and fire-fighting, non-military security work, etc.

The use of single-UAV system is very common, but using a group of small UAVs has become advantageous. Nonetheless, multi-UAV systems have some exclusive challenges and one of the most important design issues is the communication. There are many advantages of multi-UAV systems, such as

- Economical: The installation and maintenance cost of small UAVs are much less than that of a large UAV [1].

- Flexibility: Single UAV have limited coverage area, hence coverage rate is low [2]. However, multi-UAV systems can adapt to the situation easily.

- Continuity: If the UAV operation (operated by one UAV) fails in a mission, it cannot proceed. However, if a UAV goes off in a multi-UAV system, the operation can be survived through other UAVs.

- Faster: It has been shown that the missions can be completed faster with a higher number of UAVs [3].
- Higher accuracy: Instead of one large radar cross-section, multi-UAV systems produce very small radar cross-sections which are more accurate and crucial for military applications [4].

- Sustainable: Multi UAVs are more sustainable than single UAV system.

- Easy to solve: Multi-UAVs sometime can be solved recursively, which is much easier than single UAV system.

Multi-UAV systems have several issues. In a single-UAV system, a ground base station or a satellite is used for communication. Sometimes, communication link is established between the UAV and an airborne control system. In every case, single-UAV communication link is established between the UAV and the infrastructure. When the number of UAVs increases in the unmanned aerial systems, designing effective network architectures becomes a crucial issue.

There are some UAVs, those connect with a ground base station; others can connect to satellites, thereby realizing the UAV-to-UAV communication through the infrastructure. However, there are several design limitations with the infrastructure-based approach. First of all, each UAV must be equipped with an exclusive and complex hardware in order to communicate with a ground base station or a satellite. Reliability of the communication is the second issue. Another problem is the range restriction among the UAVs and the ground base station. If a UAV is outside the coverage area of the ground station, it becomes disconnected.

To resolve all the above mentioned issues, an alternative solution for multi-UAV systems is required to create an ad-hoc network among the UAVs, which is called FANET. In FANET, only a subset of UAVs can interconnect with the ground station or the satellite and all UAVs constitute an ad-hoc network. In this way, the UAVs can communicate with one another in addition to the ground station.

FANET is basically a special form of MANET/VANET. There are also certain differences between FANET and the traditional ad-hoc networks. Mobility degree of FANET nodes is much higher than that of MANET or VANET nodes. While typical MANET and VANET nodes are walking human beings or vehicles, respectively, FANET nodes fly in the sky. Due to high mobility of FANET nodes, the topology changes more frequently than the network topology of a typical MANET or even VANET. FANET needs peer-to-peer connections for 
synchronization and relationship of UAVs. It is required to collect data from the environment and to transmit to the command \& control center, as in wireless sensor networks [5]. Hence, FANET must support both peer-to-peer communication and converge cast traffic at the equivalent time. The distances among FANET nodes are much higher than in MANETs or VANETs [6]; so higher range of communication is needed. Multi-UAV systems may include different types of sensors, and each sensor may require different data distribution approaches.

There exist a few studies on UAV networks [7]-[11]. In [7], authors discussed Unmanned Aircraft System (UAS) where wireless communication is performed through IEEE $802.11 \mathrm{~b} / \mathrm{g}$ and Dynamic Source Routing (DSR) protocol was used as the routing protocol. In [8], a net-centric communication process is described with full command and control architecture for a heterogeneous unmanned aircraft system (in a small topology). In [9], authors discussed several networking issues related to delay-tolerant mobile ad-hoc network architecture. However, none of these works have provided a comprehensive survey of the routing issues of FANET networks. A survey [10] was performed on Flying ad-hoc network where FANET application scenario are discussed. It also discusses the FANET Communication protocols that consist of Physical, MAC, Network, Transport and Cross-layer architectures. FANET network layer has been discussed briefly in [11] where it is proposed that with a small modification on the routing protocols (used for VANET and MANET), they can be used in FANET architecture. However, none of these works [10], [11] have provided a comprehensive survey of the routing issues of FANET networks.

The main objective of this paper is to explain FANET as a distinct ad hoc network family and to introduce unique challenges, design constraints and routing issues in FANETs.

The contributions of this paper are (i) presenting different challenges and issues of FANET design, (ii) classifying existing routing protocols for FANET, and (iii) critically analyzing and comparing them based on various performance criteria.

Our comparative analysis will help network engineers in choosing appropriate routing protocols based on the specific scenario where the FANET will be deployed.

The rest of the paper is organized as follows. In Section II, we present several FANET designing issues. In Section III, we provide an extensive evaluation of the existing FANET routing protocols. In Section IV, we provide a comparative study among the six basic protocols of FANETs, followed by a discussion of open problems for FANET research in Section V. Finally, Section VI has the concluding remarks.

\section{FANET DESIGN ISSUES}

FANET is a new form of MANET where the nodes are UAVs. So a single-UAV systems cannot form a FANET, and valid only for multi-UAV systems. Again all multi-UAV systems do not form a FANET. UAV communication should create an ad-hoc network between UAVs to create FANET. Therefore, if the communication between UAVs fully relies on UAV-to-infrastructure links, it cannot be classified as a FANET [10].

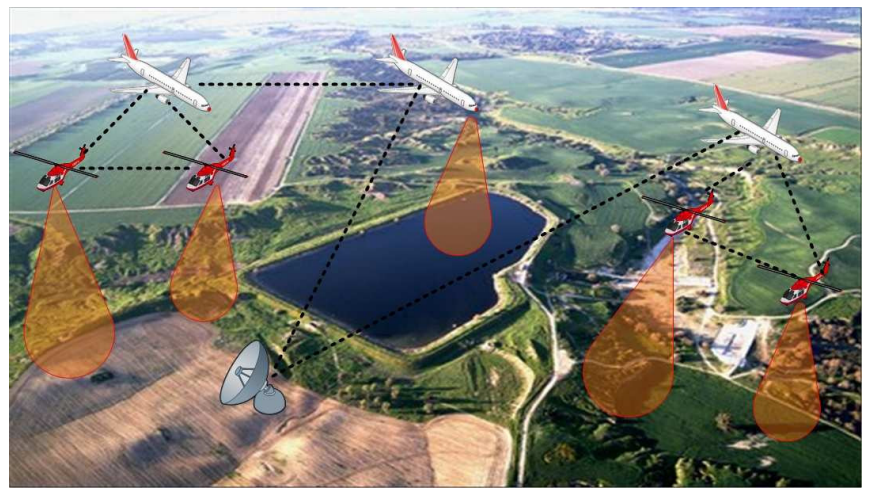

Fig. 1. A FANET scenery of multi-UAV systems.

In Fig. 1, a detailed multi-UAV system is shown. There are several area where FANET linked researches are studied under dissimilar names. Like, aerial robot team [12], aerial sensor network [13]-[15], but exact FANET base study is less interest in this topics. UAV ad hoc network [16] is totally a unique topic, which is thoroughly associated to FANETs. In fact, there is no major change between the existing UAV ad-hoc network researches and the above FANET definition. However, FANET term instantly prompts that it is a specialized form of MANET and VANET. This is why it is called Flying Ad-Hoc Network, FANET.

FANET vs. traditional ad-hoc networks:

Wireless ad hoc networks are categorized permitting to their application, positioning, communication and assignment intentions. By characterization, FANET is a form of MANET, and there are many mutual design thoughts for MANET and FANET. FANET can also be classified as a subset of VANET, which is also a subgroup of MANET.

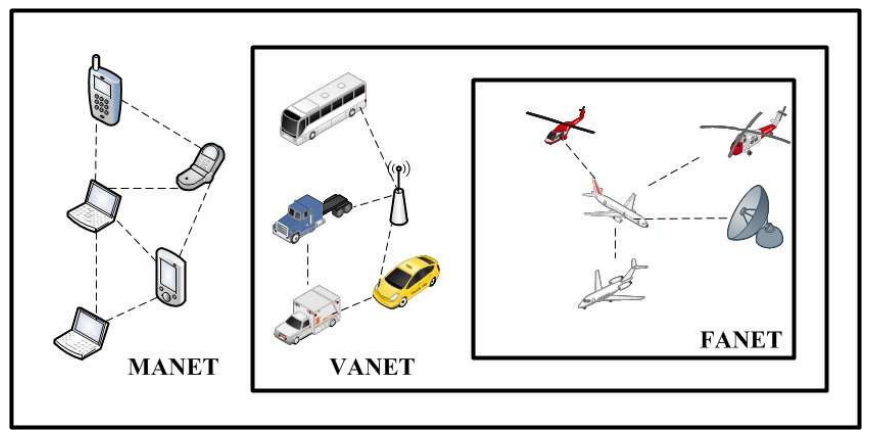

Fig. 2. MANET, VANET and FANET.

This affiliation is shown in Fig. 2. FANET shares common characteristics with these networks, and it also has some unique design challenges. Table I presents a comparison among FANET, VANET and MANET. In the following subsections, the differences among FANET and the existing wireless ad hoc networks are explained in details. 
TABLE I

COMPARISON AMONG FANET, VANET AND MANET

\begin{tabular}{|c|c|c|c|}
\hline $\begin{array}{ll}\text { Ad-Hoc network Types } \\
\text { Criteria }\end{array}$ & FANET & VANET & MANET \\
\hline Node mobility & High compactness & Medium compactness & Low compactness \\
\hline Mobility model & $\begin{array}{l}\text { Usually predetermined, but special } \\
\text { mobility models for independent } \\
\text { multi-UAV systems }\end{array}$ & Steady & Arbitrary \\
\hline Node density & Low thickness & Medium thickness & Low thickness \\
\hline Topology change & Rapid and speedy & Average speed & Slow and steady \\
\hline Radio propagation model & $\begin{array}{l}\text { High above the ground level,LoS } \\
\text { (Line of Sight) is accessible for } \\
\text { most of the cases }\end{array}$ & $\begin{array}{l}\text { Close to ground, LoS is not acces- } \\
\text { sible for all cases }\end{array}$ & $\begin{array}{l}\text { Very close to ground, LoS is not } \\
\text { accessible for all cases }\end{array}$ \\
\hline $\begin{array}{l}\text { Power consumption and network } \\
\text { lifetime }\end{array}$ & $\begin{array}{l}\text { Needed for mini UAVs, but not } \\
\text { needed for small UAVs }\end{array}$ & Not needed & Need of energy efcient protocols \\
\hline Computational power & Very big & Average & Limited \\
\hline Localization & GPS, AGPS, DGPS, IMU & GPS, AGPS, DGPS & GPS \\
\hline
\end{tabular}

\section{A. Node mobility}

Node mobility issues are the most significant difference between FANET and the other ad hoc networks. MANET node movement is comparatively slow when it is compared to VANET. In FANET, the nodes mobility degree is much higher than in the VANET and MANET. According to [6], a UAV has a speed of $30-460 \mathrm{~km} / \mathrm{h}$, and this situation results in several challenging communication design problems [17].

\section{B. Mobility model}

MANET nodes move on a definite territory, VANET nodes move on the highways, and FANET nodes fly in the sky. In multi-UAV systems, the flight plan is not fixed, if a multi-UAV system uses predefined flight plans it may not be successful, because of the environmental deviations or operation updates, the flight plan may need to be recalculated.

\section{Node density}

Node density is defined as the average number of nodes in a unit area. FANET nodes are normally spread in the sky, and the distance between UAVs can be several kilometers even for small multi-UAV systems. As a result of this, FANET node density is much lower than in the MANET and VANET.

\section{Topology change}

Due to higher mobility degree, FANET topology changes more regularly than MANET and VANET topology. When a UAV fails, the links that the UAV has been involved in also failed and it results in a topology update. Another factor that affects the FANET topology is the link outages. Because of the UAV schedules and variations of FANET node distances, link quality changes very quickly, and it also causes link outages and topology changes [18].

\section{E. Radio propagation model}

FANET and the other ad hoc network operating environments affect the radio propagation characteristics. MANET and VANET nodes are very close to the ground, and in many cases, there is no line of-sight between the sender and the receiver. Radio signals are mostly affected by the geographic structure. Again, FANET nodes those are away from the ground can be driven remotely and in maximum case; there is a line-of sight between UAVs [10].

\section{F. Power consumption and network lifetime}

Developing energy efficient communication protocols is a major part to increase the network lifetime. Particularly, while the battery-powered computing devices in MANETs; system developers have to pay extra attention to the energy efficient communication protocols. However, FANET communication hardware is powered by the energy source of the UAV. This means FANET communication hardware has no power resource problem as like in MANET.

\section{G. Computational power}

MANET nodes are battery powered small computers such as laptops, PDAs and smart phones. Because of the size and energy constraints, the nodes have only limited computational power. On the other hand VANETs and FANETs support devices with high computational power.

\section{H. Localization}

In MANET, GPS is generally used to receive the coordinates of a mobile communication terminal, and maximum time, GPS is enough to regulate the location of the nodes. In VANET, for a navigation-grade GPS receiver, there is about $10-15 \mathrm{~m}$ accuracy, which can be satisfactory for route guidance. Because of the high velocity and dissimilar mobility models of multiUAV systems, FANET needs highly accurate localization data with smaller time intervals. GPS provides position information at one second interval, and it may not be adequate for certain FANET protocols.

\section{FANET NETWORKING PROTOCOLS}

There exists many routing protocols for wireless and adhoc networks, such as pre-computed routing, dynamic source routing, on demand routing, cluster based routing, flooding, etc. FANET is a sub-class of VANET and MANET networks. Therefore, MANET routing protocols are initially chosen and tested for FANET. Due to the UAV-specific issues, such as, 
rapid changes in link quality, most of these protocols are not directly applicable for FANET networks. Hence, in order to implement this new networking model, some definite adhoc networking protocols have been implemented and some previous ones have been adapted. These protocols can be classified into six major categories:

- Static protocols, having fixed routing tables (no need to refresh these tables).

- Proactive protocols, have periodically refreshed routing tables.

- Reactive protocols (also called on-demand protocols) discover paths for messages on demand.

- Hybrid protocols that use both proactive and reactive protocols.

- Position/Geographic Based protocols that use position or area coverage.

- Hierarchical protocols that use hierarchy model for routing.

With the help of all these routing protocols, a FANET can actively discover new paths among the communicating nodes.

\section{A. Static Routing Protocols}

In static routing protocol, a routing table is calculated and uploaded to UAV nodes before an assignment, and cannot be updated during the operation; this is why it is called static. UAVs in this protocol have a constant topology [19]. Here every node communicates with a limited numbers of UAVs or ground stations, and it only preserves their information. In case of a failure (of a UAV or ground station), for updating the tables, it is essential to wait until the assignment is finished. As a result there are no fault tolerant approach for dynamic environments in static routing protocols.

1) Data Centric Routing: UAVs wireless communication support one to many data transmission which is similar to oneto-one data transmission [20], [21]. This method is selected when the data is requested by a number of nodes, and data distribution is done by on-demand algorithms. Data-centric routing is a favorable model of routing mechanism and can be adjusted for FANET [22], [23].

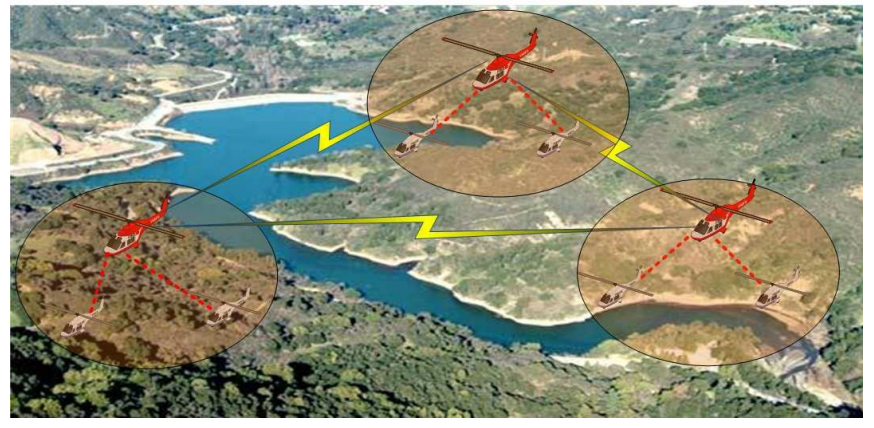

Fig. 3. Data centric routing model in FANET.

Data demand and gathering are done by data attributes instead of sender and receiver nodes' IDs. As shown in Fig. 3, this model is usually skillful with cluster infrastructure.
In this model, the consumer node (either ground node or a UAV) broadcasts queries (such as "get video of area $\mathrm{X}$ if there is a change of more than \% 3") as contribution message in order to collect particular data from a precise area. Routing is done with respect to the content of data. Data aggregation algorithms may be used for energy efficient data broadcasting. This routing executes three scopes of decoupling:

- Space decoupling: Communicating parties can be anywhere.

- Time decoupling: Data can be transmitted to the subscribers instantly or later.

- Flow decoupling: Delivery can be accomplished constantly.

This model can be chosen when the system contains a small number of UAVs on a planned path, which involves minimum assistance.

2) Multi-Level Hierarchical Routing: Organizing UAV networks hierarchically a number of clusters needs to operate in different mission areas, as shown in Fig. 4. Each cluster has a cluster head $(\mathrm{CH})$, which will represent the whole cluster; this separate cluster can perform different activities. Each $\mathrm{CH}$ is in connection with the upper/lower layers (ground stations, UAVs, satellites, etc.) directly or indirectly. To broadcast data and control info to other UAVs in the cluster, $\mathrm{CH}$ should be in direct communication range of other UAVs in cluster.

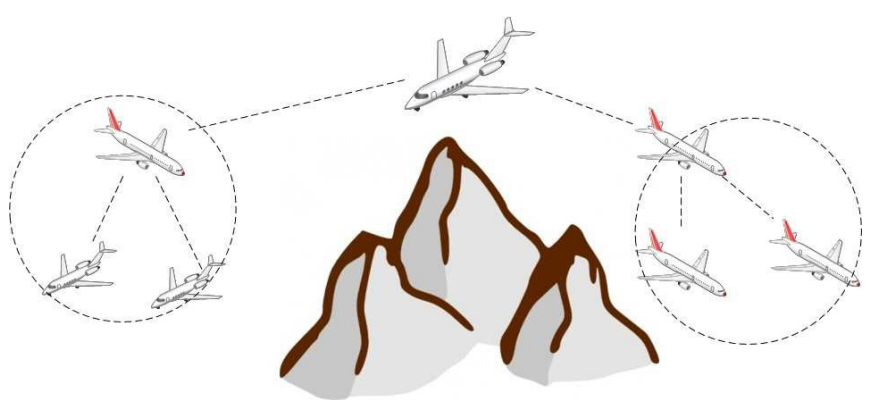

Fig. 4. Multi-Level Hierarchical routing model in FANET

This model is better if UAVs are controlled in changed swarms, the mission area is huge, and several UAVs are used in the network.

3) Load Carry and Deliver Routing: In this model, a UAV loads data from a ground node; then the data is being carried to the destination by flying; and at the end the data reached to the destination ground node.

The main objectives of load carry and deliver routing is to maximize throughput and increase the security. But the main drawback of this protocol is whenever the distance of communicating parts growth, the transmission delay becomes tremendously huge and unendurable. To solve this problem multi-UAVs system can be developed so that it decreases transmission delay as well as the distance among UAVs.

\section{B. Proactive Routing Protocols}

Proactive routing protocols (PRP) use tables to store all the routing in the network. The main advantage of proactive 
routing is that it stores the latest information of the routes; therefore, it is easy to choose a route from the sender to the receiver, as a result transmission delay can be minimized. But, there are some disadvantages of this protocol.

First, there are lot of messages are being exchange between nodes, therefore bandwidth optimization is not possible. For this reason PRPs are not suitable for highly mobile and/or larger networks. Second, when the topology change or connection failure occurs, PRP shows a slow reaction.

1) Directional Optimized Link State Routing: This protocol is based on the well known Optimized Link State Routing Protocol (OLSR) [24]. One of the most important factors that affect the OLSR performance is to select multipoint relay (MPR) nodes. The sender node selects a set of MPR nodes so that the MPR nodes can cover two hop neighbors. One of the most crucial design issues for OLSR is the number of MPRs, which effects the delay dramatically. Simulation studies [25] showed that DOLSR can reduce the number of MPRs with directional antennas.

2) Destination Sequenced Distance Vector: This protocol mainly uses the Bellman Ford algorithm with slight modifications for ad hoc networks. In DSDV, each node saves a routing table (with sequence number) for all other nodes, not just for the neighbor nodes [26]. Whenever the topology of the network changes, these changes are circulated by the protocol to update devices. To eliminate routing-loops and to identify the latest route, DSDV uses sequence numbers, which are allocated by destination nodes. The route which has higher sequence number is selected.

The main advantages of DSDV are easy algorithm and the usage of sequence numbers, which guaranteed the protocol to be loop free. Again, it has some drawbacks. For an upto date routing table, each node periodically broadcast routing table updates, which brings overhead to the network.

3) Topology Broadcast based on Reverse-Path Forwarding: This protocol use Dynamic Source Routing (DSR) [27]. The main advantage to choose DSR is its reactive configuration. The source tries to find a path to a destination, only if it has data to send. Main drawback of this protocol is the topology is unstable when the nodes are highly mobile.

\section{Reactive Routing Protocols}

Reactive Routing Protocol (RRP) can be referred as on demand routing protocol. If there is no connection between two nodes, there is no need to calculate a route between them. The concept RRP is came to overcome the overhead problem of PRP.

There are two different messages in this protocol: Route_Request messages and Route_Reply messages. Route_Request messages are created and transmitted by flooding to the network by the source node, and the destination node responses to this message with a Route_Reply message. RRP is bandwidth efficient, because there is no periodic messaging. Main drawback is it takes long time to find the route; as a result high latency may occur.
1) Dynamic Source Routing: Dynamic Source Routing (DSR) is designed for wireless mesh networks [28]. In DSR, the source node broadcasts a route request message to its neighbor nodes. In the entire communication route, there can be many route request messages. So, to avoid mix-up, the source node added a distinctive request_id. If the source node is not capable to use its present route (changes in the network topology), then the route repairs mechanism is triggered. This routing protocol was implemented by Brown et al. in [29] and they found finding a new route in UAV network with DSR can be frustrating.

2) Ad-hoc On-demand Distance Vector: Ad-hoc Ondemand Distance Vector (AODV) has almost the similar ondemand features with DSR. The only difference is routing table maintenance [30].

In DSR each node can store multiple entries in the routing table for each destination while in AODV; there is a single record for each destination. Another difference in DSR, the data packets transfer the complete path between source and the destination nodes. But in AODV, the source node only stores the next-hop information consistent to each data communication.

AODV routing protocol consists of three phases: route discovery, packet transmitting and route maintaining.

3) Time-slotted on-demand Routing: Time-slotted ondemand routing protocol is proposed in [31] for FANETs. Basically it is time-slotted version of AODV. Time-slotted on-demand protocol uses dedicated time slots in which only one node can send data packet. Although it increases the use of network bandwidth but mitigates the packet collisions and ensure packet delivery.

\section{Hybrid Routing Protocols}

To overcome the limitations of previous protocol Hybrid routing protocol (HRP) is introduced. Reactive routing protocols needs extra time to discover route and proactive routing protocols has huge overhead of control messages both can mitigate in HRP. HRP is appropriate for large networks. A network can be divided into a number of zones where intrazone routing used proactive method while inner-zone routing uses reactive method [11].

1) Zone Routing Protocol: Zone Routing Protocol (ZRP) is based on the concept of zones [32]. In this protocol, each node has a different zone. The zone is defined as the set of nodes whose minimum distance is predefined radius $R$. So, the zones of neighboring nodes intersect. The routing inside the zone is called as intra-zone routing, and it uses proactive method. If the source and destination nodes are in the same zone, the source node can start data communication instantly. When the data packets need to send outside the zone the interzone routing is used and reactive method is applied.

2) Temporarily Ordered Routing Algorithm: Temporarily Ordered Routing Algorithm (TORA) routers only preserve info about adjacent routers [33]. TORA mainly uses a reactive routing protocol but it also use some proactive protocol. It constructs and preserves a Directed Acyclic Graph (DAG) 
from the source node to the destination. TORA does not use a shortest path solution, sometime longer routes used to reduce network overhead. Each node has a parameter value termed as "height" in DAG, which is unique for each node. Data flow as a fluid from the higher nodes to lower. It is structurally loop-free because data cannot flow to the node that has higher value [11].

\section{E. Position/Geographic Based Routing Protocols}

Position-based routing needs information about the physical position of the contributing nodes in the network. Generally, each node calculates its own location through the use of GPS or some other type of positioning facilities. Position based routing is primarily motivated by two subjects, $(i)$ A position facility is used by the sender of a packet to decide the position of the destination and ( $i i)$ A forwarding approach used to forward the packets.

1) Greedy Perimeter Stateless Routing: Greedy Perimeter Stateless Routing (GPSR) is a position based protocol, have several advantages over proactive and reactive routing protocols. Shirani et al. developed a simulation framework to study the position-based routing protocols for FANETs [34]. The outcome of the study is that GPSR can be used for compactly positioned FANETs. But, reliability is the major issue of this protocol. For this another method, like face routing, can be used to achieve more reliability.

2) Geographic Position Mobility Oriented Routing: The traditional position-based protocols only depend on the location information of the nodes. But, geographic position mobility oriented routing predicts the movement of UAVs with Gaussian - Markov mobility model, and uses this information to guess the next hop.

\section{F. Hierarchical Routing Protocols}

In hierarchical routing protocols the choice of proactive and of reactive routing depends on the hierarchic level. The routing is primarily established with some proactive planned routes and then helps the request from by triggered nodes through reactive protocol at the lower levels. The main drawbacks of this protocol are: complexity and addressing scheme which response to traffic request as a result it hang the interconnecting factors.

1) Mobility prediction clustering: It operates on the dictionary of Trie-structure calculation algorithm and link termination time mobility model to guess network topology updates. In this way, it can build more constant cluster formations [35].

2) Clustering algorithm of UAV networking: It constructs the clusters on the ground, and then updates the clusters through the mission in the multi-UAV system [36].

\section{COMPARATIVE STUDY}

As mentioned earlier, there exist six basic protocols for FANET. In this section, we critically analyze and compare these basic FANET protocols. Table II presents the comparative study among these six FANET routing protocols: static, proactive, reactive, hybrid, position/geographic based and hierarchical protocols. We explain each of the comparison criteria in more details in this section:

\section{A. Main Idea}

The main idea for static protocol is routing information is fixed for a specific mission and loaded into the UAVs before the mission. Proactive protocol stores the current route information into the table. Reactive protocol is on demand protocol; when the source asks for destination route, it calculates the route. Hybrid protocol is a combination of both proactive and reactive protocols. Position/geographic protocol uses GPS or other location service to calculate the route. Hierarchical protocols uses hierarchy model to find route.

\section{B. Complexity}

For static protocol, complexity is relatively low because destination is fixed. However, for proactive, reactive and hybrid protocol, complexity is medium. In case of topology change, route finding becomes more complex in proactive protocol. For position-based protocol, finding route becomes difficult if the location service is poor. In an urban area, hierarchical protocol is useful but its setup is not so simple.

\section{Route}

In case of static protocol, route is fixed throughout the mission. For all other protocols, routes are dynamic.

\section{Topology size}

Static protocol is used for fixed mission. As a result, if the topology size is large, there is chance of topology change. Hence, static protocol is suitable for small networks. Proactive protocol is a table-driven protocol; hence, if the number of UAVs increases, their corresponding routing table entries also increases. Thus, proactive protocol is suited for small networks. For hybrid protocols, intra-zone routing is usually fixed and small sized. Position based and hierarchical protocols are used in larger network.

\section{E. Memory size}

In static protocols, the whole routing information is uploaded into the UAVs before the mission. As a result, it requires large memory space. If the number of nodes increases, the table size grows larger. Thus, proactive protocol requires larger memory. Reactive protocol is source driven; hence, when source is required to find route, it is activated, requiring less memory. Position-based protocol caches the coordinates of each UAV, thereby requireing large memory space. Hierarchical protocol uses hierarchical structure whose memory requirement is low.

\section{F. Fault tolerant}

In FANETs, mission route or topology change is a very common scenario. However, static protocols do not support this scenario. Therefore, fault tolerance is absent in this protocol. However, every other protocol has some fault tolerance. 
TABLE II

COMPARISONS AMONG THE BASIC ROUTING PROTOCOLS IN FANETS.

\begin{tabular}{|c|c|c|c|c|c|c|}
\hline $\begin{array}{lr} & \begin{array}{r}\text { Different } \\
\text { Protocol } \\
\text { Types }\end{array} \\
\text { Criteria } & \end{array}$ & Static Protocols & $\begin{array}{l}\text { Proactive Proto- } \\
\text { cols }\end{array}$ & $\begin{array}{l}\text { Reactive Proto- } \\
\text { cols }\end{array}$ & Hybrid Protocols & $\begin{array}{l}\text { Position/Geographic } \\
\text { Based Protocols }\end{array}$ & $\begin{array}{l}\text { Hierarchical Proto- } \\
\text { cols }\end{array}$ \\
\hline Main Idea & $\begin{array}{l}\text { Static routing ta- } \\
\text { ble }\end{array}$ & $\begin{array}{ll}\text { Table driven } \\
\text { protocols }\end{array}$ & $\begin{array}{l}\text { On demand pro- } \\
\text { tocol }\end{array}$ & $\begin{array}{l}\text { Combination of } \\
\text { proactive and } \\
\text { reactive protocols }\end{array}$ & $\begin{array}{l}\text { Position-based pro- } \\
\text { tocol }\end{array}$ & $\begin{array}{l}\text { Protocol } \\
\text { maintained through } \\
\text { hierarchy }\end{array}$ \\
\hline Complexity & Low & Medium & Average & Average & High & High \\
\hline Route & Static & Dynamic & Dynamic & Dynamic & Dynamic & Dynamic \\
\hline Memory size & High & High & Low & Medium & High & Low \\
\hline Fault tolerant & Absent & Present & Present & Mostly present & Present & Present \\
\hline Bandwidth Utilization & Maximum & Minimum & Maximum & Medium & Minimum & Maximum \\
\hline Convergence Time & Fast & Slow & Mostly fast & Average & Average & Average \\
\hline Signalling Overhead & Absent & Present & Present & Present & Present & Present \\
\hline Communication Latency & Low & Low & High & High & Low & High \\
\hline Mission Failure Rate & High & Low & Low & Very low & Very low & Very low \\
\hline
\end{tabular}

\section{G. Bandwidth utilization}

Static protocols are used in small network where topology is fixed; as a result, bandwidth utilization is high in this protocol. Proactive protocols have to send hello messages periodically in the network. Therefore, this protocol requires more bandwidth. Reactive protocols are source driven, requiring less bandwidth. For hybrid protocols, bandwidth utilization is medium. Position based protocol send source location as extra information; hence, bandwidth consumption rate is higher. Hierarchical protocols use limited bandwidth as each UAV is connected to upper level UAVs.

\section{H. Convergence time}

In the static protocol, destination is predetermined. As a result route finding time is minimal. Proactive protocol searches the destination node after each topology change, resulting in larger converge time. Reactive protocols usually find route much faster but if topology changes, this protocol takes more time than normal case. Hybrid, position based and hierarchical protocols usually take average time to converge.

\section{Signalling overhead}

Other than static protocols, each protocol (proactive, reactive, hybrid, position based and hierarchical) have signaling overheads, such as hello message in proactive protocols, route request and route reply message in reactive protocols, etc.

\section{J. Communication latency}

Static, proactive and position based protocols have low communication latency since the distance between the UAVs in these protocols is small. Reactive, hybrid and hierarchical protocols have higher latency because UAV-to-UAV and UAVto-ground station distance is much higher in these protocols.

\section{K. Mission failure rate}

Topology and route change are common phenomena in FANETs.Other than static protocols, each protocol has backup strategy for topology change. Only static protocols do not have any strategy when topology or route changes, as a result mission failure rate is very high in this protocol.

\section{Popularity}

Static protocols is not fault tolerant and position based protocols need extra mechanism to find the positions of the UAVs. This is why, these two protocols are least popular. Rest of the protocols are much more popular.

\section{Applications}

Static protocols are used in missions where mission objective and topology are fixed. Hierarchical protocols are mostly used in military operations where communication is difficult. Previously, most of the protocols were used in military operations. However, use of UAVs have increased day by day. As a result, many civil operations are now conducted by multiUAVs systems. For this reason, all the protocols are being modified so that these protocols can be used in normal and civil operations.

\section{Open Research Problems}

Existing MANET and VANET routing protocols cannot satisfy all the FANET routing requirements. Therefore, routing is one of the most important and challenging issues for FANETs. In this section, we list a few open research issues regarding routing in flying ad hoc networks.

\section{A. P2P UAV communications}

In FANET, movements of UAVs are very fast, resulting in very rapid network topology change. Hence, data routings among the UAVs are challenging. The routing protocols should 
be capable of updating routing tables dynamically. Peer-topeer communication is crucial for cooperative synchronization and collision anticipation of multi-UAV systems. FANET can collect information from the environment as in wireless sensor networks, which is a different traffic configuration. Developing a peer-to-peer communication and converge cast traffic can be an attractive topic in FANETs. Data centric routing for FANETs is another encouraging approach which is still unexplored.

\section{B. Regulations for civilian UAVs}

The uses of UAVs are increasing day by day and now it has become a part of most of countries national airspace system. However, most of the existing air principles do not allow UAV operations in civil airspace. This is the biggest obstruction to the development of Unmanned Arial Systems in civilian areas. As a result, distinctive rules and guidelines to integrate UAV flights into the national airspace need to be deployed urgently.

\section{Robust FANET algorithms}

In a large area mission and multi-UAV operations, dynamic changes (such as addition / deletion of UAVs, fixed and dynamic threats, etc.) can occur. Therefore, robust algorithms with dynamic route adjustments are compulsory to coordinate the fleets of UAVs. It is essential for FANET to support qualities of services (QoS) so that it can protect against some predetermined service performance constraints, such as delay, bandwidth, jitter, packet loss, etc.

\section{UAV placement}

The sizes of mini-UAVs are small and they carry limited payloads, for example single radar, infrared camera, thermal camera, image sensor, etc. Therefore, different sensors can be merging-up with different UAVs; or one UAV can be integrated with a thermal camera and another with image sensor. Regarding this, UAV placement to reduce energy consumption is still an open issue.

\section{E. FANET standardization}

FANET uses various wireless communication bands, such as, VHF, UHF, L-Band, C-band, Ku-Band, etc. [11] which are also used in different application areas, such as GSM networks, satellite communications. For reducing congestion problem, FANETs require standardization. FANET should connect to integrate with a Global Information Grid (GIG) as one of the main information platforms to increase its efficiency.

\section{F. Coordination of UAVs with Manned aircraft}

In the future, flights of UAVs with other manned aircraft are likely to increase. Coordination of these two will ensure the destruction of opponent aircraft with minimal losses. Therefore, the association of UAVs and manned aircraft should be in a networked environment.

\section{CONCLUSION}

Unmanned Aerial Vehicles have promising role in a large operation zone with complicated missions. For the region that are reasonably isolated from the ground and to accomplish complex tasks, UAVs require cooperation with one another and need a quick and easy deploying network system. MultiUAV system reduces the operation accomplishment time and increases reliability of the system for airborne operations when compared to a single-UAV system. To apply networking in non-LOS, urban, aggressive, and noisy environment, multiUAV system is very effective and accurate.

Communication is one of the most challenging issues for multi-UAV systems. In this paper, ad hoc networks among the UAVs, i.e, FANETs are surveyed along with its key challenges compared to traditional ad hoc networks. The existing routing protocols for FANETs are classified into six major categories which are then critically analyzed and compared based on various performance criteria. Finally, we list several open research issues related to FANET routing protocols to inspire researchers work on these open problems.

\section{REFERENCES}

[1] H. Chao, Y. Cao, and Y. Chen, "Autopilots for small fixed-wing unmanned air vehicles: a survey," International Conference on Mechatronics and Automation, 2007 (ICMA 2007), pp. 3144-3149, 2007.

[2] B. Morse, C. Engh, and M. Goodrich, "Uav video coverage quality maps and prioritized indexing for wilderness search and rescue," Proceedings of the 5th ACM/IEEE International Conference on HumanRobot Interaction, HRI 10, Piscataway, NJ, USA, vol. 3, pp. 227-234, 2010.

[3] E. Yanmaz, C. Costanzo, C. Bettstetter, and W. Elmenreich, "A discrete stochastic process for coverage analysis of autonomous uav networks," Proceedings of IEEE Globecom-WiUAV, IEEE, 2010.

[4] L. To, A. Bati, and D. Hilliard, "Radar cross-section measurements of small unmanned air vehicle systems in non-cooperative field environments," 3rd European Conference on Antennas and Propagation, 2009 (EuCAP 2009), IEEE, pp. 3637-3641, 2009.

[5] M. Rieke, T. Foerster, and A. Broering, "Unmanned aerial vehicles as mobile multi-platforms," The 14th AGILE International Conference on Geographic Information Science,Utrecht, Netherlands, 18-21 April 2011.

[6] J. Clapper, J. Young, J. Cartwright, and J. Grimes, "Unmanned systems roadmap," Tech. rep., Dept. of Defense, pp. 2007-2032.

[7] T. Brown, B. Argrow, E. Frew, C. Dixon, D. Henkel, J. Elston, and H. Gates, "Experiments Using Small Unmanned Aircraft to Augment a Mobile Ad Hoc Network," ISBN-13: 9780521895842, pp. 179-199.

[8] J. Elston, E. Frew, D. Lawrence, P. Gray, and B. Argrow, "Net-centric communication and control for a heterogeneous unmanned aircraft system," Journal of Intelligent and Robotic Systems, vol. 56(1-2), pp. 199-232, 2009.

[9] E. Frew and T. Brown, "Networking issues for small unmanned aircraft systems," Journal of Intelligent and Robotics Systems, vol. 54 (1-3), pp. 21-37, 2009

[10] I. Bekmezci, O. K. Sahingoz, and S. Temel, "Flying ad-hoc networks (FANETs): A survey," Elsevier, Ad Hoc Networks 11, pp. 1254-1270, 2013

[11] O. K. Sahingoz, "(FANETs): Concepts and challenges," Springer J Intell Robot System, vol. 74, pp. 513-527, 2014

[12] S. Cameron, S. Hailes, S. Julier, S. McClean, G. Parr, N. Trigoni, M. Ahmed, G. McPhillips, R. de Nardi, J. Nie, A. Symington, L. Teacy, and S.Waharte, "SUAAVE: Combining aerial robots and wireless networking," 25th Bristol International UAV Systems Conference, 2010.

[13] A. Purohit and P. Zhang, "SensorFly: a controlled-mobile aerial sensor network," in ACM,7th ACM Conference on Embedded Networked Sensor Systems, SenSys '09, New York, NY, USA, 2009, pp. 327-328. 
[14] M. Akbas and D. Turgut, "APAWSAN: actor positioning for aerial wireless sensor and actor networks," in 36th Conference on Local Computer Networks, LCN '11, IEEE Computer Society, Washington, DC, USA, 2011, pp. 563-570.

[15] J. Allred, A. Hasan, S. Panichsakul, W. Pisano, P. Gray, J. Huang, R. Han, D. Lawrence, and K. Mohseni, "Sensorock: an airborne wireless sensor network of micro-air vehicles," ACM, 5 th International Conference on Embedded Networked Sensor Systems, pp. 117-119, 2007.

[16] T. Brown, S. Doshi, S. Jadhav, and J. Himmelstein, "Test bed for a wireless network on small UAVs," AIAA 3rd Unmanned Unlimited Technical Conference, pp. 20-23, 2004.

[17] Z. Han, A. Swindlehurst, and K. Liu, "Optimization of MANET connectivity via smart deployment/movement of unmanned air vehicle," IEEE Transactions on Vehicular Technology, vol. 58, pp. 3533-3546, 2009.

[18] E. Yanmaz, R. Kuschnig, and C. Bettstetter, "Channel measurements over 802.11a-based UAV-to-ground links," GLOBECOM Wi-UAV Workshop, pp. 1280-1284, 2011.

[19] A.Franchi, C.Secchi, M.Ryll, H. Bulthoff, and P.R.Giordano, "Shared control: Balancing autonomy and human assistance with a group of quad rotor UAVs," IEEE Robot. Auto Mag, vol. 19 (3), pp. 57-58, 2012.

[20] J.Ko, A.Mahajan, and R.Sengupta, "A network-centric UAV organization for search and pursuit operations," IEEE Aerospace Conference, pp. 2697-2713, 2002.

[21] J.Lopez, P.Royo, E.Pastor, C.Barrado, and E. maria, "A middleware architecture for unmanned aircraft avionics," ACM/IFIP/ USENIX International Conference on Middleware companion (MC 07), 2007.

[22] E. D. Jong, "Flexible data-centric UAV platform eases mission adaptation," White paper:http://www.rti.com/whitepapers/RTI-Data-DrivenApproach-to-UAV.pdf, 3 Aug 2013.

[23] A. A. Koller and E.N.Johnson, "Design, implementation, and integration of a publish/subscribe-like multi- UAV communication architecture," AIAA Modelling and Simulation Technologies Conference and Exhibit, pp. 1-17, 2005.

[24] T. Clausen and P. Jacquet, "Optimized link state routing protocol (OLSR),” RFC 3626 (Experimental), October 2003.

[25] A. Alshabtat, L. Dong, J. Li, and F. Yang, "Low latency routing algorithm for unmanned aerial vehicles ad-hoc networks," International Journal of Electrical and Computer Engineering, vol. 6 (1), pp. 48-54, 2010.

[26] D. Jung and P. Tsiotras, "Inertial attitude and position reference system development for a small uav," 26th AIAA Aeroacoustics Conference, 2007.

[27] D. Johnson and D. Maltz, "Dynamic source routing in ad hoc wireless networks," Mobile Computing, The Kluwer International Series in Engineering and Computer Science, Springer, US, vol. 353, pp. 153 $181,1996$.

[28] D.B.Johnson and D.A.Maltz, "Dynamic source routing in ad hoc wireless networks," Kluwer Academic Publishers, pp. 153-181, 1996.

[29] T.X.Brown, B.Argrow, C.Dixon, S.Doshi, R.G.Thekkekunel, and D.Henkel, "Ad-hoc UAV ground network (AUGNet)," 3rd AIAA Unmanned Unlimited Technical Conference, pp. 29-39, 2004.

[30] S.Murthy and J. L. Aceves, "An efficient routing protocol for wireless networks," ACM Mobile Networks and Applications, pp. 183-197, 1996.

[31] J. Forsmann, R. Hiromoto, and J. Svoboda, "A time-slotted on-demand routing protocol for mobile ad-hoc unmanned vehicle systems," SPIE $6561,2007$.

[32] Z.J.Haas and M.R.Pearlman, Zone Routing Protocol (ZRP) a hybrid framework for routing in ad-hoc networks. Addison-Wesley, 2001, vol. 1.

[33] V. Park and S.Corson, "Temporarily-ordered routing algorithm (TORA)," Version 1. Internet draft:IETF MANET working group. http://tools.ietf.org/html/ draft-ietf-manet-tora-spec-04, 3 Aug 2013.

[34] R. Shirani, M. St-Hilaire, T. Kunz, Y. Zhou, J. Li, and L. Lamont, "The performance of greedy geographic forwarding in unmanned aeronautical ad-hoc networks," in 2011 Ninth Annual Communication Networks and Services Research Conference, CNSR '11, IEEE Computer Society, Washington, DC, USA, 2011, pp. 161-166.

[35] C. Zang and S. Zang, "Mobility prediction clustering algorithm for UAV networking," in GLOBECOM Workshops, IEEE, 2011, pp. 1158-1161.

[36] L. Kesheng, Z. Jun, and Z. Tao, "The clustering algorithm of UAV networking in near-space," in 8th International Symposium on Antennas, Propagation and EM Theory,(ISAPE 2008), 2008, pp. 1550-1553. 